Geistige Eigentumsrechte, was steht für die Entwicklungsländer auf dem spiel?

\title{
Vision und Dialog : die Zielsetzungen der Schweiz im globalen System des geistigen Eigentums
}

\section{T. Thu-Lang Tran}

\section{(2) OpenEdition}

\section{Journals}

Electronic version

URL: http://journals.openedition.org/sjep/682

DOI: $10.4000 /$ sjep.682

ISSN: 1663-9677

\section{Publisher}

Institut de hautes études internationales et du développement

\section{Printed version}

Date of publication: 1 avril 1998

Number of pages: 47-64

ISSN: $1660-5926$

\section{Electronic reference}

T. Thu-Lang Tran, «Vision und Dialog : die Zielsetzungen der Schweiz im globalen System des geistigen Eigentums », Schweizerisches Jahrbuch für Entwicklungspolitik [Online], 17 | 1998, Online erschienen am: 01 August 2012, abgerufen am 08 September 2020. URL : http:// journals.openedition.org/sjep/682 ; DOI : https://doi.org/10.4000/sjep.682 


\title{
VISION UND DIALOG: DIE ZIELSETZUNGEN DER SCHWEIZ IM GLOBALEN SYSTEM DES GEISTIGEN EIGENTUMS
}

\author{
T. THU LANG TRAN*
}

\section{EINFÜHRUNG}

1987 - 1997 : Zehn Jahre sind seit dem Beginn der Uruguay-Runde verstrichen. Pessimisten sahen damals in dieser Verhandlungsrunde lediglich ein weiteres Unterfangen, das von vornherein zum Scheitern verurteilt war: Die Bestrebungen der internationalen Organisationen, die entweder eine Verstärkung des patentrechtlichen Schutzes erreichen (wie die Weltorganisation für geistiges Eigentum, WIPO) oder den Bereich des geistigen Eigentums auf andere Weise an sich reissen wollten (wie die UNCTAD) schienen dafür Beweis genug. Heute, im Jahr 1997, sind die Welthandelsorganisation (WTO) und deren Abkommen über handelsbezogene Aspekte des geistigen Eigentums (TRIPSAbkommen) ' nicht mehr wegzudenkende Bestandteile des wirtschaftlichen und politischen Lebens. Die Geschwindigkeit, mit der dieses neue multilaterale Handelssystem von den Staaten und besonders von den Entwicklungsländern akzeptiert wurde, und die Eile, mit der andere Staaten ihren Beitritt zur Organisation vorantreiben, sind bemerkenswert. Das kommerzielle Interesse allein reicht als Erklärung nicht aus. Das GATT/WTO bietet den Staaten ein Diskussionsforum, welches sich strukturell von den spezialisierten Agenturen der UNO abhebt. Seine unterschiedlichen Verhandlungsmechanismen erklären zumindest teilweise das derzeitige reibungslose Funktionieren der Organisation und haben nicht zuletzt zum Erfolg der Uruguay-Runde beigetragen, denn dies war der Moment, in dem sämtliche Möglichkeiten der spezifischen GATT/WTO-Verhandlungstechnik voll ausgeschöpft werden konnten.

In Erwartung der zweiten Ministerkonferenz der WTO, die im Mai 1998 in Genf stattfinden soll, werden zahlreiche Berichte über die bisherigen Tätigkeiten, aber auch Prognosen über die nächste «built-in agenda» und über neue Verhandlungsbereiche wie Investitionen oder Wettbewerb erstellt. Auf dem Gebiet des geistigen Eigentums hat der TRIPS-Rat die Anfangsphase der Arbeiten bereits überwunden und erledigt Angelegenheiten wie z.B. die Notifizierung von Gesetzen und Regelungen, die keine Diskussion hervorrufen, auf rein «technische» Weise. Die Arbeitssitzungen verlaufen jedoch keineswegs routinemässig, denn die Überprüfung der nationalen Gesetze der Industrieländer führt immer wieder zu offenen, mehr oder weniger gütlich ausgetragenen Konfrontationen, besonders zwischen den USA und den europäischen Staaten. Der TRIPS-Rat ist bei seiner Tätigkeit an Termine gebunden, die ein relativ hohes Arbeitstempo diktieren: In naher Zukunft werden die Verhandlungen über die geographischen Angaben für Weine und Spirituosen anlaufen, 1999 werden die

\footnotetext{
* Leitung der Sektion Internationale Angelegenheiten, Eidgenössisches Institut für Geistiges Eigentum, Bern. Die in diesem Artikel geäusserten Meinungen sind nur für den Verfasser verbindlich.

1. SS 0.632.20, S. 367-402, TRIPS : Abkürzung für Trade-related Aspects of Intellectual Property Rights.
} 
Vorschriften über die Patentfähigkeit lebender Organismen und im Jahr 2000 die Anwendbarkeit des Prinzips der «Nichtverletzung» untersucht werden müssen. Ebenfalls im Jahr 2000 werden die Übergangsfristen für eine Reihe von Entwicklungs- und Reformländer ablaufen. Dann müssen wie schon in den vergangenen zwei Jahren die nationalen Gesetze und Regelungen dieser Länder überprüft werden.

Wenn Bilanz gezogen und Prognosen formuliert werden, stellt sich auch die Frage, welche Bereiche für die Schweiz künftig von besonderer Bedeutung sein werden. Ein Blick zurück auf die zahlreichen wertvollen Erfahrungen der Vergangenheit dürfte bei der Betrachtung der Zukunft, die einerseits ein grosses Potential birgt und in der andererseits alle Szenarien möglich sind, von grossem Nutzen sein. Im folgenden wird ein Abriss über die Position der Schweiz und ihrer Verhandlungspartner während der Uruguay-Runde vermittelt, wobei besonders auch auf die Standpunkte der Entwicklungsländer eingegangen wird. Danach wird ein Überblick über die Entwicklungszusammenarbeit im Bereich des geistigen Eigentums geboten. In diesem Zusammenhang soll auch der Entwurf eines neuartigen bilateralen Abkommens behandelt werden. Abschliessend werden einige Überlegungen über die Bedeutung des geistigen Eigentums für die Entwicklungsländer angesichts der zunehmenden Globalisierung angestellt, wobei die Rolle der Schweiz besondere Aufmerksamkeit erhält.

\section{DIE SCHWEIZ UND DAS GEISTIGE EIGENTUM IM ALLGEMEINEN}

Die Schweiz verfügt weder über ein wettbewerbsfähiges Lohnniveau noch über attraktive Bodenschätze. Wenn die schweizerischen Exportgüter und -dienstleistungen einen Wertzuwachs aufweisen sollen, so kann dieser nur auf dem Wege der Innovation oder des technologischen Vorsprungs oder über irgendeine andere Form der Kreativität erzielt werden. Dabei stösst man jedoch an ein Paradox, das typisch ist für ein immaterielles Gut: Je höher der innovative Wert eines Produktes ist, desto häufiger wird dieses Produkt nachgeahmt. Sobald es aber imitiert worden ist, verkleinert sich sein Handelswert für den Eigentümer, oft sogar in einem drastischen Umfang. Der so verursachte Schaden schlägt sich jedoch nicht nur in den Umsatzzahlen nieder, sondern er kann sich auch auf den Ruf eines Unternehmens auswirken, wenn das Imitat von schlechter Qualität ist und der Konsument das Produkt mit einem Unternehmen assoziiert. Dies kann beispielsweise bei Medikamenten oder bei Ersatzteilen für den Flugzeugbau zu besonders schweren Schädigungen führen. Von der Nachahmung sind neben den Patenten auch Markenzeichen, gewerbliche Muster sowie geographische Angaben betroffen. Bei einem Patent erleidet der Erfinder oder das Unternehmen, welches in Forschung und Entwicklung ( $\mathrm{F} \& \mathrm{E}$ ) investiert hat, einen Verlust. Bei den Markenzeichen liegen die Dinge so, dass die Investitionen und Marketingaktivitäten zur Assoziierung einer Marke mit einem Produkt und mit dessen Hersteller immer kostspieliger werden. Dasselbe gilt für gewerbliche Muster. Der unrechtmässige Gebrauch geographischer Angaben führt nicht nur zu einer Schwächung des Identifikations- und Marketingpotentials, sondern auch zur Erosion des Erbes einer Region oder eines Landes.

Das für Wirtschaft und Handel äusserst wichtige Gebiet des geistigen Eigentums war zu Beginn der Uruguay-Runde längst kein Neuland mehr. In diesem 
Bereich existieren internationale Übereinkommen, die teilweise über 100 Jahre alt sind und laufend aktualisiert werden, um mit der technologischen und der intellektuellen Entwicklung Schritt halten zu können. ${ }^{2}$ Die Schweiz ist Gründungsmitglied der durch diese Übereinkommen geschaffenen Organisationen oder Institutionen. Nach so vielen Jahrzehnten scheint es nicht verwunderlich, dass das geistige Eigentum mittlerweile Teil einer gewissen «Kultur» oder Weltanschauung geworden ist, in der es nur natürlich erscheint, die erfinderische Tätigkeit fortzusetzen oder Erfindungen zu verbessern und dafür belohnt zu werden. Die Schweiz gilt als eines der Länder mit der weltweit höchsten Patentdichte. Ihr Name steht für Qualität (z.B. «Swiss made») und besitzt eine überzeugende Verkaufskraft, und sie ist in hohem Masse abhängig vom Export ihrer Güter und Dienstleistungen mit hohem Mehrwert. Dies ist auch der Grund dafür, weshalb die Verhandlungen über das geistige Eigentum im Rahmen des GATT für die Schweiz von besonderem Interesse waren. Gleichzeitig war sie sich als kleines Land bewusst, dass diesbezügliche Bemühungen auf multilateraler Ebene nur dann zum Erfolg führen würden, wenn sie eine möglichst breite internationale Unterstützung, insbesondere die der Entwicklungsländer und der beiden Supermächte, geniessen.

Die schweizerische Exportwirtschaft wird in sämtlichen Bereichen, in denen sie führend ist, mit dem Problem der Nachahmung konfrontiert. Mit den Fortschritten in Kommunikation und Technologie lassen sich Nachahmungen immer einfacher bewerkstelligen, und die Bekämpfung dieses Phänomens gestaltet sich dementsprechend schwieriger. Ein nicht vorhandener oder unzureichender Schutz des geistigen Eigentums wirkt sich dissuasiv auf die Schweizer Unternehmen und deren Strategie aus - schliesslich würden sie wohl kaum in einem Land Investitionen tätigen, wenn diese Investitionen zur Herstellung eines Gutes aufgrund des fehlenden rechtlichen Rahmens gefährdet sind. Dasselbe gilt natürlich für Entwicklungsländer und für Länder im Übergang zur Marktwirtschaft. Allerdings kann auch ein zu strenger Schutz oder das Bestehen stark voneinander abweichender Schutzsysteme zu Verzerrungen führen. ${ }^{3}$ Die Frage des geistigen Eigentums stellt nicht nur die Anliegen der Industrieländer denjenigen der Entwicklungsländer gegenüber, sondern auch die Interessen der Industrieländer untereinander. ${ }^{4}$ Auch wenn die Möglichkeiten des GATT zur Beseitigung von Streitigkeiten auf dem Gebiet des geistigen Eigentums zu Beginn hauptsächlich auf das Problem der Nachahmungen in den Entwicklungsländern ausgerichtet war, darf die Bedeutung dieses Nord-Nord-Gegensatzes nicht vernachlässigt werden. ${ }^{5}$ Diese Tatsache dürfte zur endgültigen Klärung eines alten Missverständnisses und zum besseren Verständnis einiger Bestimmungen des TRIPS-Abkommens beitragen.

2. Pariser Verbandsübereinkunft zum Schutze des gewerblichen Eigentums (1883), Berner Übereinkunft zum Schutz von Werken der Literatur und Kunst (1886).

3. z.B. das Ersterfinderprinzip oder die Kosten für das Enforcement-Verfahren (Verfahren zur Geltendmachung der Rechte am geistigen Eigentum) in den USA.

4. Die Revision der Pariser Verbandsübereinkunft zu Beginn der 80er Jahre wirkte sich nicht nur auf die Entwicklungsländer (Zwangslizenzen), sondern auch auf die Industrieländer aus: Der Schutz der geographischen Angaben beispielsweise führte zu weitreichenden Divergenzen zwischen manchen europäischen Ländern, darunter die Schweiz, und anderen Industrieländern (USA, Kanada, Australien und Neuseeland).

5. Dieser Aspekt ist von besonderer Wichtigkeit, was sich auch beim Überprüfungsprozess der nationalen Gesetzgebungen der Industrieländer immer wieder bestätigt, besonders bei den Patenten, den geographischen Angaben und beim Enforcement. 


\section{DIE URUGUAY-RUNDE, DIE TRIPS, DIE SCHWEIZ UND DIE ENTWICKLUNGSLÄNDER}

\subsection{VON PUNTA DEL ESTE BIS 1991}

Bei der Festlegung des Tagungsortes für die Ministerkonferenz war die Wahl Uruguays keineswegs ein Zufall. Die Verhandlungspartner hatten den Wunsch geäussert, die Entwicklungsländer möchten bei der achten Verhandlungsrunde eine aktivere Rolle spielen als in der Vergangenheit. Die 1986 in Punta del Este verabschiedete Erklärung legte nicht nur die Verhandlungsziele fest, sondern enthielt auch ein Mandat zur Ausweitung des Geltungsbereiches des Allgemeinen Zoll- und Handelsabkommens auf neue Gebiete wie Dienstleistungen, Investitionen und geistiges Eigentum. ${ }^{6}$

Während der Anfangsphase, zwischen 1987 und 1989, waren die Verhandlungen durch zwei entgegengesetzte Ansätze geprägt. Diese waren allerdings zu Beginn der Uruguay-Runde nicht neu, sondern im Laufe der 70er und 80er Jahre von der UNCTAD oder der WIPO bereits wiederholt vertreten worden. Der erste, hauptsächlich von den Industrieländern unterstützte Ansatz tendierte in Richtung eines international geltenden Systems, das es den Unternehmen erlauben sollte, zumindest einen angemessenen Teil der Investitionskosten sowie der Kosten für F \& E zu decken. Die Unternehmen erachteten diese Frage als umso dringlicher, als sich die Konkurrenz im Zuge der Globalisierung verschärfte. Der zweite Ansatz, der von den Entwicklungsländern vertreten wurde, berief sich auf das Prinzip, wonach das Schutzniveau des geistigen Eigentums ein Gleichgewicht zwischen Kosten und Nutzen des Schutzes darstellen sollte. Die Entwicklungsländer vertraten die Ansicht, ein internationales System mit eingeschränktem Geltungsbereich würde ihnen die Möglichkeit geben, ihre eigene Entwicklung voranzutreiben, und machten sogar das Recht geltend, sich bei der wirtschaftlichen Entwicklung zumindest zu Beginn auf Nachahmungen zu stützen. Aber selbst unter den Befürwortern dieses zweiten Ansatzes gingen die Ansichten auseinander, was die Situation schwieriger gestaltete und sich auf den weiteren Verlauf der Gespräche auswirkte.

Die Industrieländer strebten ausserdem eine Ausweitung der Verhandlungen auf die Festsetzung von Regeln zum geistigen Eigentumsrecht an. Die Entwicklungsländer hingegen vertraten die Ansicht, das GATT müsse sich auf die Aushandlung internationaler Normen zur Bekämpfung der Nachahmung beschränken, denn die Aufstellung materiell- und verfahrensrechtlicher Normen sei Aufgabe der WIPO. Aber auch die Industrieländer waren zu Beginn weit von einer gemeinsamen Position entfernt. Deshalb musste parallel zum offiziellen Verhandlungsprozess in der zuständigen Verhandlungsgruppe (GN 11) der Uruguay-Runde in einem informellen Rahmen, nämlich in der Gruppe der «Freunde des geistigen Eigentums», unter den Industrieländern ein Konsens gesucht werden. Die Schweiz nahm zusammen mit allen anderen Industrieländern an den Diskussionen dieser Gruppe aktiv teil. Angesichts der grossen Bedeutung des geistigen Eigentums für die wirtschaftliche Entwicklung der Schweiz war ihr Engagement mit demjenigen der grossen Han-

6. Für Details zum Verhandlungsablauf siehe Bibliographie: GATT-Botschaft 1, op. cit., Girard, op. cit., Croone, op. cit.

7. Otten, op. cit., S. 72. («in essence the static costs from limitations on access to technology and other forms of intellectual property vs the dynamic benefits from the incentive to R \& D and other forms of creativity ».) 
delspartner durchaus vergleichbar. Dieses Verfahren wurde anschliessend auf die Gruppe der Entwicklungsländer ausgeweitet. Teilnehmer waren die Tigerstaaten Asiens, einige lateinamerikanische Staaten und ein osteuropäisches Land. Gleichzeitig wurden innerhalb der GN 11 grosse Anstrengungen unternommen, um präzise Informationen über die nationalen und internationalen Systeme zusammenzutragen und die Probleme und Vorbehalte der Länder zu identifizieren. Die eigentlichen Verhandlungen begannen aber erst 1989. Sowohl von den Industrie- als auch von den Entwicklungsländern gingen zahlreiche Beiträge ein. Der Stein kam jedoch erst im März 1990 richtig ins Rollen, als die EG, gefolgt von den USA, der Schweiz, Japan und einer Gruppe von 14 Entwicklungsländern, die ersten konkreten Vorschläge einbrachten. Diese Vorschläge stellten eine ausreichende «kritische Masse» dar, die es dem Vorsitzenden der Verhandlungsgruppe und dem Sekretariat erlaubten, ein «informelles» plurilaterales Verfahren mit einer beschränkten Anzahl Staaten einzuleiten. Im Anschluss daran konnte der Vorsitzende die Verhandlungsgruppe (d.h. alle Vertragsparteien) informieren. Die ersten Arbeitspapiere, bei denen es sich um einfache Aufstellungen ( « composite texts») handelte, wurden dann im weiteren Verlauf der Verhandlungen bereinigt, um die Vielzahl der Vorschläge auf einige gemeinsame Nenner zu reduzieren. Die Verhandlungen selbst in der sogenannten « $10+10 »-$ Gruppe waren zäh und intensiv, aber auch erstaunlich offen. Danach wurde der Text von «Gruppen variabler Geometrie » die sich aufgrund von gemeinsamen Interessen und Bündnissen bildeten - weiter überarbeitet, um möglichst viele der eingeklammerten Passagen zu eliminieren. Bei den Verhandlungen traten aber auch weitreichende Divergenzen zwischen den Industrieländern zutage. Diese Divergenzen waren bereits in anderen Gremien zum Vorschein gekommen und hatten dort zu einer Lähmung der Diskussion geführt. Dennoch gelang es den Verhandlungsparteien, sich im Dezember 1991 auf einen Vertragstext zu einigen, der sowohl von der Struktur als auch vom Inhalt her vollständig war. Danach wurden an dem Text nur noch geringfügige Änderungen vorgenommen, bevor er am 15. Dezember 1993 mit dem Entwurf für die Schlussakte verabschiedet wurde. Die Schweiz hat während der gesamten Runde, besonders aber zwischen 1987 und 1991, zur Verteidigung ihrer Interessen eine aktive Rolle gespielt.

\subsection{POSITIONEN DER PARTNER DER SCHWEIZ}

Bei der Betrachtung der TRIPS-Verhandlungen darf das globale Umfeld der Uruguay-Runde nicht ausser acht gelassen werden. Während die USA und die Entwicklungsländer diametral entgegengesetzte Verhandlungspositionen vertraten, nahm die EG eine etwas nuanciertere Haltung ein. Dafür gab es mehrere Gründe: Zum einen musste sie abwarten, bis sich unter ihren Mitgliedsstaaten ein Konsens herausgebildet hatte. Dies war eine komplexe Prozedur, schliesslich handelte es sich um interne multilaterale Verhandlungen. Zum andern verfügte sie nur in jenen Bereichen über ausreichend Spielraum, in denen sie selbst Bestimmungen erlassen konnte, und die Fragen zur Landwirtschaft und Textilindustrie trugen nicht gerade zu einer Festigung ihrer Position bei $^{8}-$ dies war

8. Die EG hatte zu diesem Zeitpunkt Bestimmungen über die Zollbehandlung von Nachahmungen (Markenzeichen) und Piratenware (Urheberrechte) erlassen. Zudem bestanden bereits Regelungen über geographische Bezeichnungen, über Weine und Spirituosen sowie in neueren Gebieten (Topographie integrierter Schaltkreise etc.). Es sei daran erinnert, dass die Schweiz in landwirtschaftlichen Fragen ebenfalls in der Defensive stand. 
zumindest der Eindruck, den ein aussenstehender Beobachter erhalten konnte. Das in den UNO-Sonderorganisationen praktizierte Prinzip der Regionalgruppen kam überdies im GATT nicht zur Anwendung. Auch die Gruppe der Entwicklungsländer mit ihrer Vielzahl an Anliegen bildete längst keinen homogenen Block. Indien und Argentinien repräsentierten eine unnachgiebige Front, die auf einem minimalen Abkommen über Nachahmung und Piraterie bestand. Die ost- und südostasiatischen Länder sowie einige lateinamerikanische Staaten zeigten sich anderen Vorschlägen gegenüber eher offen, vorausgesetzt, sie konnten Zugeständnisse in anderen Bereichen wie z.B. beim Zugang zum amerikanischen oder europäischen Markt oder einen ausreichenden «Deal» im Bereich der Landwirtschaft aushandeln. Wie sich im weiteren Verlauf der Verhandlungen herausstellte, nahm auch Indien je nach Thema eine differenziertere Haltung ein ${ }^{9}$, so zum Beispiel bei der Verteidigung der geographischen Angaben bei landwirtschaftlichen Gütern oder verarbeiteten Produkten, als sich Indien der schweizerischen Position anschloss. Im Laufe der Uruguay-Runde sah sich die Schweiz als kleines Land mehrmals gezwungen, derartige Koalitionen mit variabler Geometrie einzugehen, mit jeweils einigen wenigen Industrieländern sowie einer gewissen Anzahl Entwicklungsländer als Partner.

\subsection{POSITION DER SCHWEIZ WÄHREND DEN TRIPS-VERHANDLUNGEN}

Als Land mit einem der strengsten Schutzsysteme der Welt ${ }^{10}$ hatte sich die Schweiz folgende Prioritäten gesetzt: Gleichbehandlung für schweizerische Unternehmen und Unternehmen der grossen Handelspartner aus den Reihen der Entwicklungsländer ${ }^{11}$; verbesserter Schutz von Erfindungen (auf sämtlichen technologischen Gebieten) durch Patente, Markenzeichen, gewerbliche Muster (besonders in der Uhren- und Textilindustrie), geographische Angaben (auch für industrielle Produkte und Dienstleistungen) und Geschäfts- und Fabrikationsgeheimnisse (Know-how); bessere Durchsetzung der Rechte am geistigen Eigentum (enforcement); Transparenz; Beilegung und Vermeidung von Streitigkeiten, und - last but not least - Komplementarität mit den Bemühungen der WIPO und anderen involvierten internationalen Organisationen. Im Bereich der Urheberrechte und der verwandten Schutzrechte sowie auf dem Gebiet der Topographie integrierter Schaltkreise waren die Handelsinteressen der Schweiz geringer als beispielsweise jene der EG oder der USA. Um jedoch die Kohärenz mit den Gesetzesvorlagen nicht zu beeinträchtigen, die damals erarbeitet wurden (Notwendigkeit, die Haltung interessierter Kreise in Sachen Urhebberrechte und benachbarte Schutzrechte $\mathrm{zu}$ vertreten) ${ }^{12}$, und angesichts dieses für die

9. Indien lehnte eine Verbesserung des Patentsystems kategorisch ab. Das Land hatte aber beachtliche Interessen im Bereich des Urheberrechts, schliesslich exportiert Indien Filme und nimmt auf dem Gebiet der neuen Technologien, wie z.B. Computerprogramme, eine starke Position ein (Indien war der Berner Übereinkunft [Urheberrechte] beigetreten, aber nicht der Pariser Verbandsübereinkunft [gewerbliches Eigentum einschliesslich Patente]).

10. Die Schweiz ist nicht nur den meisten von der WIPO verwalteten Übereinkommen beigetreten, sondern auch dem Europäischen Patentübereinkommen von 1973, einem Vertragswerk von grosser regionaler Bedeutung (Mitgliedsstaaten: EG-Staaten, Liechtenstein, Monaco und die Schweiz).

11. Die Schweiz war selber Opfer eines flagranten Falls von Diskriminierung geworden und befürwortete deshalb eine Meistbegünstigungsklausel im Bereich des geistigen Eigentums. (Bei dem erwähnten Fall von Diskriminierung handelt es sich um die von Südkorea praktizierte "pipeline protection» (retroaktiver Patentschutz im pharmazeutischen Bereich), der ausschliesslich amerikanischen Unternehmen und später Firmen aus der EG gewährt wurde, aber nicht den südkoreanischen Unternehmen, was gegen das Prinzip der Inländerbehandlung verstiess. Es dauerte sechs Jahre, bis die Schweiz dieselbe Behandlung erhielt.)

12. Dies gilt besonders für die kulturellen Aspekte oder die geistigen Urheberrechte, ein Thema, in dem eine grosse Kluft zwischen europäischen und amerikanischen Ansichten herrschte. 


\section{PATENTFÄHIGER GEgenstand}

Vorschlag der Schweiz im Rahmen der Uruguay-Runde

MTN/GNG/NG11/W/73, 14. Mai 1990 (Originaltext: englisch)

«Artikel 229

1) Patente werden für jede Art von Erfindungen, ob es sich um Erzeugnisse oder Verfahren handelt, erteilt, die möglicherweise gewerblich anwendbar und neu sind und auf einer erfinderischen Tätigkeit beruhen.

2) Patente werden für Erfindungen aus allen Bereichen der Technik erteilt.

3) Die Erteilung eines Patents für eine Erfindung kann verweigert werden, falls die Veröffentlichung der Erfindung oder ihre gewerbliche Anwendung gegen die öffentliche Ordnung verstösst, das grundlegende Prinzip der menschlichen Würde verletzt oder mit den allgemein akzeptierten guten Sitten nicht vereinbar ist.

4) Die VERTRAGSPARTEIEN können für Pflanzensorten neben dem Schutz durch Patente ein Schutzsystem sui generis vorsehen.

5) Patente werden nach dem Erstanmelderprinzip erteilt."

Verhandlungsergebnis (geltende Bestimmung des TRIPS-Abkommens)

"Artikel 27 Patentfähiger Gegenstand

1. Vorbehaltlich der Absätze 2 und 3 werden Patente für Erfindungen, ob es sich um Erzeugnisse oder Verfahren handelt, auf allen Gebieten der Technik erteilt, sofern sie neu sind, auf einer erfinderischen Tätigkeit beruhen und gewerblich anwendbar sind? Vorbehaltlich des Artikels 65 Absatz 4, des Artikels 70 Absatz 8 und des Absatzes 3 dieses Artikels erfolgt die Erteilung von Patenten und die Ausübung von Patentrechten unabhängig vom Ort der Erfindung, vom Gebiet der Technik oder davon, ob die Erzeugnisse eingeführt oder im Land selbst hergestellt werden.

2. Die Mitglieder können Erfindungen von der Patentierbarkeit ausschliessen, wenn die Verhinderung ihrer gewerblichen Verwertung in ihrem Hoheitsgebiet zum Schutz der öffentlichen Ordnung oder der guten Sitten einschliesslich des Schutzes des Lebens oder der Gesundheit von Menschen, Tieren oder Pflanzen oder zur Vermeidung einer schweren Schädigung der Umwelt notwendig ist, sofern der Ausschluss nicht allein deshalb vorgenommen wird, weil das Landesrecht die Verwertung verbietet.

3. Die Mitglieder können von der Patentierbarkeit auch ausschliessen:

a) diagnostische, therapeutische und chirurgische Verfahren für die Behandlung von Menschen oder Tieren;

b) Pflanzen und Tiere mit Ausnahme von Mikroorganismen sowie im wesentlichen biologische Verfahren zur Züchtung von Pflanzen oder Tieren mit Ausnahme von nichtbiologischen und mikrobiologischen Verfahren. Die Mitglieder sehen jedoch den Schutz von Pflanzensorten entweder durch Patente oder durch ein wirksames System sui generis oder durch eine Verbindung beider vor. Die Bestimmungen dieses Buchstabens werden vier Jahre nach Inkrafttreten des WTO-Abkommens überprüft.

7. (Fussnote zu Art. 27) Für die Zwecke dieses Art. können die Mitglieder die Ausdrücke " erfinderische Tätigkeit " und "gewerblich anwendbar " als Synonyme der Ausdrücke "nicht naheliegend " beziehungsweise "nützlich» betrachten.

(Anmerkung der Autorin: Artikel 65.4 gibt den Entwicklungsländern die Möglichkeit, eine zusätzliche Übergangsfrist von 5 Jahren zu beantragen, um Bestimmungen zum patentrechtlichen Schutz von Erfindungen in jenen Gebieten der Technik einzuführen, für die ein solcher Schutz zum Zeitpunkt des Inkrafttretens des TRIPS-Abkommens (1. Januar 1995) nicht vorgesehen war. Dies ergibt insgesamt eine Übergangsfrist von zehn Jahren. Die davon am häufigsten betroffenen Sektoren sind die Pharmazeutik und die Agrochemie. Artikel 70.3 regelt die Nichtretroaktivität des TRIPS-Abkommens für Gegenstände, die am Tag der Anwendung des TRIPS-Abkommens durch ein bestimmtes Land (1.1.2000 oder je nach Fall 1.1.2005) Allgemeingut geworden sind.) 
Industrie zukunftsträchtigen Bereichs (insbesondere für schweizerische KMU im High-Tech-Sektor wie z.B. Software und Topographie integrierter Schaltkreise) war sie gezwungen, sich in diesen Aspekten ebenso stark zu engagieren. Schliesslich waren der elektronische, mechanische, chemische und pharmazeutische Sektor von der Durchsetzung der Rechte, vom Prinzip des «government use» und vom Geltungsbereich der Rechte gleichermassen betroffen. Die Schweiz gilt in weiten Kreisen der internationalen Öffentlichkeit als Synonym für die chemische und pharmazeutische Industrie und wurde oft als einer der grössten Verfechter des Patents für pharmazeutische und biotechnologische Produkte bezeichnet. Ihr Engagement bei den Verhandlungen, besonders in Fragen zu umstrittenen technologischen Bereichen, wurde des öfteren kritisiert. Wiederholt wurde das Argument vorgebracht, die Schweiz habe nicht das «Recht», von den Entwicklungsländern einen Patentschutz für Produkte zu verlangen, der in der Schweiz selbst erst im Jahr 1978 eingeführt worden war. Objektiv gesehen sprechen verschiedene Tatsachen gegen dieses Argument: Erstens existierten in der Schweiz ein weitreichender Schutz für Produktionsverfahren und effiziente juristische Verfahren und Rechtsmittel. Problematisch wird der Verfahrensschutz dann, wenn das Verfahren nachgeahmt wird und das Land, in welchem die Nachahmung stattfindet, über kein effizientes und wirksames rechtliches Instrumentarium zu ihrer Bekämpfung verfügt. Zweitens war die Schweiz bei der Einführung von Massnahmen zum Schutz von Produkten nicht allein, sondern andere europäische Länder wie Deutschland und Österreich verfolgten ähnliche Ziele. Drittens ist der Vergleich nicht zutreffend, denn zwischen 1973, als das Europäische Patentübereinkommen verabschiedet wurde, und dem Beginn der Uruguay-Runde 1987 machte der technologische Bereich eine rasante Entwicklung durch. Dieser technologische Fortschritt erleichterte die Nachahmung von Erzeugnissen, gleichzeitig aber bedingte er für die innovierenden Unternehmen ständig wachsende Investitionen zur Entwicklung leistungsfähigerer Produkte. Zudem fand eine Globalisierung der Handelsbeziehungen statt. Vor diesem Hintergrund setzte sich die Schweiz für eine differenzierte Behandlung der Entwicklungsländer ein und befürwortete die Gewährung von Übergangszeiten, die allerdings in Anbetracht der erzielten technologischen Fortschritte verkürzt werden könnten. Ausserdem stellte sich mehr und mehr heraus, dass ein Land, das sich vom «Mainstream» abheben will, sich ins Abseits manövriert. Diesen Tatsachen kann nur begegnet werden, indem Übergangsfristen zur wirtschaftlichen Restrukturierung zugestanden werden und während dieser Übergangsfrist technische Unterstützung gewährt wird, die auf die jeweiligen Bedürfnisse zugeschnitten ist.

\subsection{DIE HALTUNG DER SCHWEIZ GEGENÜBER DEM PATENT}

Das Patentrecht war unbestritten ein bedeutendes Anliegen der Schweiz. Gleichzeitig stellte dieses Thema einen der heikelsten Punkte in den Beziehungen zwischen den Industrieländern dar und führte zu den wohl emotionalsten Debatten in den Nord-Süd-Verhandlungen. Dazu kommt, dass die grundlegenden Probleme wie Armut, Ernährung und öffentliches Gesundheitswesen in den Entwicklungsländern, von denen sich jeder einzelne betroffen fühlt, zu einer noch dramatischeren Atmosphäre in den Verhandlungen führten. Die Spannungen, die die Diskussionen umgaben, brauchen also nicht weiter beschrieben zu 
werden. Rückblickend gewinnt man den Eindruck, dass sämtliche Punkte, die weder in der UNCTAD noch in der WIPO geregelt werden konnten, den TRIPS-Verhandlungen aufgebürdet wurden. Es existieren mehrere Punkte, die in der Pariser Übereinkunft unvollständig oder gar nicht geregelt sind: Kriterien für die Patentfähigkeit einer Erfindung (Neuheit, erfinderische Tätigkeit, gewerblicher Nutzen), Kategorien für den Ausschluss von der Patentfähigkeit, Schutzdauer, Definition der Rechte, die sich aus einem Patent ergeben, Beweislast im Falle einer Verletzung eines Verfahrenspatents sowie diesbezügliche Ausnahmen, Zwangslizenzen, klare Bedingungen für die Gewährung von Zwangslizenzen und der «government use ». ${ }^{13}$

Im folgenden soll in erster Linie auf einige Punkte eingegangen werden, die auch im Jahr 1998 aktuell sind:

Bei ihren 1990 gemachten Vorschlägen zu den Patenten übernahm die Schweiz die damals geltenden Bestimmungen des europäischen und schweizerischen Rechts, einschliesslich einiger Änderungsvorschläge, die den Positionen anderer europäischer Länder gerecht wurden (siehe Kasten). Zu erwähnen ist in diesem Zusammenhang, dass die Schweiz, zur grossen Überraschung der anderen Industrieländer, unter den Motiven für den Ausschluss von der Patentfähigkeit auch das "grundlegende Prinzip der menschlichen Würde» ${ }^{14}$ nannte. Im Hinblick auf den Schutz von Pflanzenzüchtungen machte die Schweiz den Vorschlag, den Ländern die Möglichkeit zu bieten, neben dem patentrechtlichen Schutz ein System sui generis ${ }^{15}$ einzuführen.

Der Vorschlag der Schweiz zum Gebrauch von Patenten ohne Einverständnis des Inhabers zielte darauf $a b$, präzise Bedingungen für die Erteilung von Zwangslizenzen zu formulieren und so die Möglichkeiten zur Gewährung solcher Lizenzen einzuschränken. Bei den Gesprächen innerhalb der UNCTAD und der WIPO war das Gebot zur Herstellung im Inland heftig umstritten. Die Schweiz vertrat die Ansicht, die Verpflichtung zur Herstellung eines Produktes in jedem Land, in welchem das Produkt patentrechtlich geschützt ist, sei in einer globalisierten Wirtschaft aufgrund der komparativen Vorteile weder realistisch noch realisierbar. Ein Verbot für ein Unternehmen, die Bedürfnisse eines Landes durch Importe zu decken, käme zudem einer mengenmässigen Einfuhr-

13. Dabei handelt es sich um die Nutzung eines Patents durch ein Unternehmen der Regierung oder durch eine Person, die für ein solches Unternehmen arbeitet, ohne Einverständnis des Patentinhabers (nach europäischem und schweizerischem Recht kommt dies einer Fälschung gleich; in Ländern mit angelsächsischer Rechtstradition, darunter gewisse Entwicklungsländer, besteht die rechtliche Möglichkeit eines solchen Gebrauchs).

14. Das Prinzip der Würde des Tieres ist angesichts der zahlreichen kulturell bedingten Wertvorstellungen unter den Vertragsparteien des GATT ein heikleres Thema, welches nach Ansicht der Schweizer Delegation durch das Konzept der «guten Sitten» abgedeckt werden sollte.

15. Die Industrieländer, besonders jene, die selber Vorschläge einbrachten, dachten dabei an das UPOV-Übereinkommen als «wirksames» Schutzsystem. Dieses wurde jedoch aus Gründen der geographischen Abdeckung nicht ausdrücklich genannt, denn der Geltungsbereich des Übereinkommens war, im Unterschied zur Pariser oder Berner Übereinkunft, zum Zeitpunkt der Verhandlungen auf die Industrieländer beschränkt. Die Entwicklungsländer hätten 1990 weder die Eingliederung des UPOV-Übereinkommens in das TRIPS-Abkommen noch die namentliche Erwähnung dieser Organisation geduldet. Zur Zeit scheint es allerdings so, als sei dieses Übereinkommen das einzige im Bereich des Schutzes von Pflanzenzüchtungen. Seit Abschluss der Uruguay-Runde traten mehrere Staaten neu dem UPOV-Übereinkommen bei, darunter einige Entwicklungs- und Reformländer sowie einige weitere Industrieländer. Die sogenannten «farmers'rights» (Rechte der Bauern) kamen während der entscheidenden Phase der UruguayRunde (1990-91) nicht zur Sprache. Sie wurden erst später bei den Gesprächen über die Artenvielfalt diskutiert, nachdem die Ausarbeitung des «Dunkel-Textes» 1991 abgeschlossen war (damals bestand auf allen Seiten wenig Hoffnung, dass darin enthaltene Fragen vor der endgültigen Verabschiedung noch einmal diskutiert werden könnten). Artikel 27.3 b des TRIPS-Abkommens verpflichtet die Staaten lediglich, einen Schutz für Pflanzensorten vorzusehen, und hindert sie keineswegs daran, auch in anderen Bereichen (z.B. in der Frage der «farmers'rights») Schutzsysteme zu errichten. 
beschränkung gleich und würde somit gegen das GATT-Abkommen verstossen. Schliesslich schlug die Schweiz auch vor, die Frage des «government use» zu regeln. Die ausschliessliche Verwendung eines Patents ohne das Einverständnis des Inhabers sei zu unterbinden, da der Patentinhaber damit seiner Rechte beraubt werde und einen materiellen Schaden erleide. Mehrere der Problemstellungen, die von der Schweiz vorgebracht wurden, sind in Artikel 31 des TRIPSAbkommens geregelt.

In ihrer Eingabe vom 14. Mai 1990 schlug die Schweiz vor, für die Entwicklungsländer und die am wenigsten entwickelten Länder (LDCs) eine Übergangszeitenregelung vorzusehen. Angesichts der Tatsache, dass die Verhandlungsthemen stark miteinander verflochten waren, sah die Schweiz davon ab, konkrete Zahlen zu nennen. Die endgültige Fassung des TRIPS-Abkommens enthält denn auch eine Übergangszeitenregelung, die nach Ansicht weiter Kreise nicht genügend differenziert ist, um sämtlichen Entwicklungsländern gerecht zu werden. Nach Ablauf dieser Übergangsfrist müssen die Entwicklungsländer ihre nationale Gesetzgebung den Erfordernissen des TRIPSAbkommens angepasst haben. Im Hinblick auf die Patente bedeutet dies, dass jene Länder, in denen noch kein Patentschutz für pharmazeutische und agrochemische Produkte besteht, bis zum 1. Januar 2000 ein solches Schutzsystem eingerichtet haben müssen. Gelingt ihnen das nicht, können sie eine zusätzliche Übergangsfrist von fünf Jahren, also bis zum 1. Januar 2005, beantragen. In Anbetracht der Zeit, die zur Gewährung der Vermarktungserlaubnis benötigt wird, bedeutet dies, dass der Inhaber eines ausländischen Patentes erst etwa ab 2015 in den vollen Genuss seiner Rechte kommt. Als Ausgleich für diesen effektiven Verlust an Schutzzeit wollten die USA die sogenante «pipeline protection» einführen, die ihnen bereits von Südkorea und anderen Staaten gewährt worden war ${ }^{16}$. Die Frage der Retroaktivität war jedoch nicht unumstritten, und es musste ein für alle Vertragsparteien akzeptabler Ausweg gefunden werden, welcher darin besteht, dass die Entwicklungsländer ein sogenanntes «Black box»-Verfahren für die Hinterlegung von Patentgesuchen einrichten müssen. Die Gesuche in der «Black box» ruhen bis zum Ende der Übergangsfrist, d.h. sie werden je nach Fall während fünf oder zehn Jahren nicht bearbeitet. Wenn das Gesuch dann bearbeitet wird, muss sich die Prüfung am Stand der Technik zur Zeit der Hinterlegung orientieren. Die Berechnung der Schutzzeit erfolgt ab dem Datum der Hinterlegung, und nicht vom Zeitpunkt der Erteilung des Patents an. Der Vorteil für den Patentinhaber, stamme er nun aus dem Entwicklungsland selbst oder aus dem Ausland, liegt darin, dass er auf diese Weise gegen Fälschungen durch Dritte vorgehen kann, die vielleicht in einem anderen Land seine Erfindung kopieren konnten und sie mangels patentrechtlicher Regelungen ausbeuten. Die Schweiz war an der Ausarbeitung dieses Kompromisses massgeblich beteiligt.

\subsection{TECHNISCHE ZUSAMMENARBEIT}

Im Hinblick auf die Pflichten der Industrieländer gegenüber den Entwicklungsländern im Bereich der technischen Unterstützung verfolgte die Schweiz von Anfang an eine sehr klare Linie, denn dieser Aspekt stellt seit jeher eine Kon-

16. Siehe dazu Fussnote Nr. 14 (supra). 
stante in der schweizerischen Aussenpolitik dar. Dies wurde auch in den Vorschlägen der Schweiz deutlich. Mit Ausnahme der ersten und der dritten Eingabe (die sich ausschliesslich mit der wirtschaftlichen Analyse der Fälschungen und des unzureichenden Schutzes bzw. mit den Schutznormen befasste) war dieser Themenkomplex in ihren Vorschlägen enthalten. Darüber hinaus befasst sich die Schweiz im Rahmen der WIPO seit langem mit Fragen zur technischen Zusammenarbeit, und da sie sich entschlossen hatte, dieses Thema auch im GATT zur Diskussion zu bringen, schien es nur zweckmässig und kohärent, sich auch dort zugunsten der technischen Zusammenarbeit einzusetzen.

\section{DIE SCHWEIZ \\ UND DIE TECHNISCHE ZUSAMMENARBEIT IM BEREICH DES GEISTIGEN EIGENTUMS}

\subsection{FAKTEN ZUR TECHNISCHEN ZUSAMMENARBEIT}

Vor der Gründung der WTO war die technische Zusammenarbeit im Bereich des geistigen Eigentums auf internationaler Ebene Angelegenheit der WIPO, des UNPD, der UNCTAD und des Europäischen Patentamtes (EPA). Die einzige zuständige Stelle innerhalb der Schweiz ist im Prinzip das Bundesamt für geistiges Eigentum (seit 1996 Eidgenössisches Institut für geistiges Eigentum, IGE), dessen Tätigkeit auf dem Komplementaritätsprinzip basiert. Aus budgetären Gründen ist sein Spielraum jedoch etwas eingeschränkt, was dazu führte, dass die Unterstützung zur Modernisierung der gesetzlichen oder gerichtlichen Rahmenbedingungen sowie Aktivitäten, die längere Aufenthalte schweizer Experten in den Entwicklungsländern bedingen, nicht mehr wahrgenommen werden können. Diese Arbeiten werden jedoch von anderen, finanziell besser gestellten Ländern bilateral fortgesetzt. Abgesehen von der Bereitstellung von Patentdokumenten (zuerst auf Papier, danach auf Mikrofilm und auf CD-Rom) ${ }^{17}$ konzentriert sich die Politik des IGE mittlerweile hauptsächlich auf die Ausbildung von Sachverständigen aus Entwicklungsländern in der Schweiz selbst und in Europa. Ein Grossteil dieser Ausbildungs- und Betreuungstätigkeit wird vom IGE aus eigenen Mitteln finanziert. Reise- und Aufenthaltskosten werden von der WIPO oder dem EPA bzw. von der DEZA übernommen. Überhaupt leistet die DEZA einen entscheidenden Beitrag zur Tätigkeit der Schweiz auf dem Gebiet des geistigen Eigentums im Rahmen der WIPO. Dank dieser finanziellen Basis (bilaterale Hilfe im multilateralen Rahmen der WIPO) war das IGE bisher stets in der Lage, jedes Jahr zwei Sachverständigen aus Entwicklungsländern oder LDCs eine relativ lange, solide und gründliche Ausbildung zu vermitteln. Aus Gründen der vorhandenen Unterrichtsmaterialien wurden französischsprachige afrikanische Länder von der Schweiz bevorzugt behandelt. ${ }^{18}$ Bei der Aus-

17. Dabei handelt es sich um wertvolle Dokumentation, die die Grundlage der Infrastruktur jedes Amtes für geistiges Eigentum bildet. Sie ermöglicht es der Öffentlichkeit bzw. den Forschern, sich über den Stand der Technik zu informieren und ihre Arbeit gegebenenfalls neu auszurichten, anstatt das Risiko einzugehen, «das Rad neu zu erfinden " und ein Patentgesuch einzureichen, das mangels Neuheit abgelehnt wird. Die Verluste durch Forschungsarbeiten an Erfindungen, die keine mehr sind, werden auf rund 30 Milliarden Franken geschätzt. Diese Verluste hätten sich vermeiden lassen, wenn sich die Unternehmen die Mühe gemacht hätten, Nachforschungen über den Stand der Technik anzustellen (Information: EPA/Agence France-Presse, 10.9.97).

18. Seit einigen Jahren wird auch das Englische als Unterrichtssprache verwendet, und das IGE kann auch in dieser Sprache Lehrgänge anbieten. 
wahl der Kandidaten kommen präzise Kriterien zur Anwendung: Sie müssen aus einem der Schwerpunktländer der Schweiz stammen (gemäss einer alljährlich von der DEZA zusammengestellten Liste), und sie müssen bereits im Dienst der Regierung arbeiten und gewisse Karrierechancen aufweisen (womit ein übermässiger «brain drain» nach Abschluss der Ausbildung vermieden werden soll). Die Sachverständigen absolvieren bei der WIPO einen einwöchigen allgemeintheoretischen Kurs. Anschliessend verbringen sie drei Wochen zur Vertiefung ihrer Kenntnisse am CEIPI (Centre d'études internationales de la propriété intellectuelle) in Strassburg. Danach folgt ein vierwöchiges Praktikum beim IGE, bei dem Registrierungstätigkeiten (Patente, Markenzeichen, gewerbliche Muster und Modelle, Topographie integrierter Schaltkreise) im Vordergrund stehen. Seit einigen Jahren wird auch ein Überblick über die Urheberrechte und die verwandten Schutzrechte vermittelt. Dieser Ausbildungsgang scheint bei den Entwicklungsländern Anklang zu finden: Immer wieder gehen Anfragen von Entwicklungsländern ein, die auf die «Schweizer Liste» der WIPO gesetzt werden möchten. Die theoretische Ausbildung über die Urheberrechte und die verwandten Schutzrechte wird vom IGE angeboten, der praktische Teil (Arbeitsweise der Gesellschaften zur Verwaltung der Urheberrechte) wird von einer dieser Gesellschaften, der SUISA, auf freiwilliger Basis im Rahmen der Zusammenarbeit mit der WIPO durchgeführt. Das IGE, ein Institut mittlerer Grösse, stellt nach Ansicht der WIPO ein nachahmenswertes Modell für die Entwicklungsländer dar. Dies ist auch der Grund für die zahlreichen Anfragen für kurze Schulungsbesuche. Das EPA, welches im Zuge der Mandate der EG seine Tätigkeit im Bereich der technischen Zusammenarbeit bedeutend ausgebaut hat, wendet sich insbesondere an das IGE, um seine eigenen Ausbildungsgänge zu ergänzen. Ausserdem wird das IGE bei der technischen Zusammenarbeit zugunsten der Länder im Übergang zur Marktwirtschaft beigezogen (in diesem Fall werden die notwendigen Gelder vom BAWI und vom EJPD zur Verfügung gestellt, in allen anderen Fällen geht die Ausbildung zu Lasten des IGE). ${ }^{19}$

Eine noch relativ unbekannte Dienstleistung im Zusammenhang mit der technischen Zusammenarbeit ist die kostenlose Durchführung von Nachforschungen über den Stand der Technik, die bei den Patentämtern der Entwicklungsländer beantragt werden kann. Eine Abfrage der Datenbanken kostet in der Regel 500 bis 1'000 Franken, wobei die Arbeitszeit des Prüfers nicht mit eingerechnet ist. Selbstverständlich ist dieser Preis für ein Unternehmen aus einem Entwicklungsland unerschwinglich. Dennoch muss sich dieses Unternehmen irgendwie davon vergewissern können, dass seine Erfindung noch nicht existiert, bevor es weitere Investitionen tätigt (die womöglich vergeblich sind, wenn die Erfindung ein bereits bestehendes Patent verletzt). In solchen Fällen schafft das IGE Abhilfe, sofern seine momentane finanzielle Situation dies erlaubt, und lässt durch seine Prüfer unentgeltliche Nachforschungen anstellen, deren Ergebnisse anschliessend über die WIPO an den Antragsteller weitergeleitet werden.

19. Folgende Länder und Organisationen haben die Ausbildungsprogramme des OFPI/IPI in Anspruch genommen: Ägypten, Albanien, Algerien, Argentinien, Belarus, Benin, Bulgarien, Burundi, Chile, China, Elfenbeinküste, Estland, Georgien, Griechenland, Indien, Indonesien, Iran, Kamerun, Kasachstan, Kolumbien, Kongo, Demokratische Republik Kongo (Zaire), Demokratische Volksrepublik Korea, Republik Korea, Kroatien, Kuba, Laos, Lettland, Litauen, Madagaskar, Mali, Marokko, Mauretanien, Republik Moldau, Niger, Peru, Philippinen, Polen, Portugal, Ruanda, Rumänien, Russland, Senegal, Singapur, Slowakei, Sri Lanka, Tansania, Thailand, Togo, Tschad, Tschechien, Ukraine, Ungarn, Vereinigte Arabische Emirate, Vietnam, OAPI (Organisation Africaine pour la Propriété Industrielle). 
Durch das Inkrafttreten des TRIPS-Abkommens haben sich die Umstände stark verändert. Zwar hat die WIPO einen Teil ihres «gesetzgeberischen Monopols » verloren $^{20}$, aber sie hat, entgegen der Erwartungen gewisser Kreise, dank der Unterstützung zahlreicher Länder und insbesondere der Schweiz ihre Stellung behaupten können. Das TRIPS-Abkommen hat nämlich neue Bedürfnisse geschaffen, welche die WTO allein nicht wahrnehmen kann und die der WIPO neuen Auftrieb verliehen haben. Gemäss Artikel 68 des Abkommens ist der TRIPS-Rat verpflichtet, innerhalb eines Jahres nach seiner ersten Sitzung unter Beiziehung der WIPO geeignete Massnahmen auszuarbeiten, die die künftige Zusammenarbeit mit den Organen dieser Institution regeln sollen. Darüber hinaus verpflichtet Artikel 67 des TRIPS-Abkommen ausdrücklich die Industrieländer zur technischen und finanziellen Zusammenarbeit mit den Entwicklungsländern und den LDCs. Die WTO verfügt jedoch nicht über ausreichende finanzielle Mittel, um die Bedürfnisse an technischer Zusammenarbeit selbst abzudecken, zumal es sich dabei um ein sehr weitreichendes Gebiet handelt. Am 22. Dezember 1995 schlossen die WTO und die WIPO ein Abkommen über die Verwaltung der nationalen Gesetzessammlungen (einschliesslich deren Übersetzung ins Französische und Englische, welche die Entwicklungsländer nicht selber finanzieren können), über die technisch-juristische Unterstützung und die technische Zusammenarbeit. Dieses Abkommen ist von grösserer Bedeutung, als gemeinhin angenommen wird, denn da die meisten Länder Mitglieder beider Organisationen sind, ergibt sich ein bemerkenswerter Synergieeffekt. Das TRIPS-Abkommen wurde zu einem festen Bestandteil der von der WIPO gewährten technisch-juristischen Unterstützung. Das neue Zweijahresprogramm und das Budget der WIPO für die Periode 1998-2000 betonen die Notwendigkeit, die technische Zusammenarbeit zugunsten der Entwicklungsländer aufzustocken, denn nur so sind diese in der Lage, den im TRIPS-Abkommen vorgesehenen Termin im Jahr 2000 einzuhalten ${ }^{21}$. Gemeinsam ist man stark: Dieses Motto bewahrheitet sich auch hier. Denn in einer Zeit, in der die öffentlichen Gelder knapper werden, ist es unumgänglich, mit vereinten Kräften vorzugehen und die Bemühungen aufeinander abzustimmen. Dieser Gedanke wird auch von der Schweiz in ihrer Politik der technischen Zusammenarbeit verfolgt.

Zwischen 1995 und 1996 bestanden einige Entwicklungsländer im Rahmen der Tagungen des TRIPS-Rates wiederholt auf der Verpflichtung der Industrieländer zur technischen Zusammenarbeit. Gemäss einer Entscheidung des Rates müssen diese periodisch Berichte über ihre diesbezügliche Tätigkeit einreichen. ${ }^{22}$ In

20. Dieser Verlust ist relativ, denn die Komplementarität auf «gesetzgeberischem » Gebiet funktioniert durchaus zufriedenstellend, was durch die neuesten Verträge im Bereich der Urheberrechte und der verwandten Schutzrechte, deren Schutz in manchen Bereichen über die Anforderungen des TRIPS-Abkommens hinausgeht, bestätigt wird. Die Schweiz unterstützt diese Bemühungen aus der Überzeugung heraus, dass das Recht weiterentwickelt und den WTO-Mitgliedsstaaten, insbesondere den Entwicklungsländern und Reformstaaten, genügend Zeit gegeben werden muss, um die zur Umsetzung des TRIPS-Abkommens notwendigen Strukturanpassungen durchzuführen.

21. «Most developing countries are due to fulfil the requirements of the TRIPS Agreement by January 1, 2000, immediately upon conclusion of this biennum. This generates a basic imperative for WIPO's development cooperation activities in the 1998-1999 biennum, given the key role it is expected to play in supporting national planning and implementation of legislative and administrative development to meet these requirements. " (WIPO-Dokument, im Druck).

22. Wie die anderen Länder hat auch die Schweiz seither drei solche Berichte eingereicht, die hauptsächlich auf die Tätigkeit des IGE eingehen (Dokumente IP/C/W/12/Add. 3 vom 13.9.95, IP/C/W/34/Add. 2 vom 17.9.96 und IP/C/W/77/Add. 3 vom 30.9.97). Diese Dokumente werden, sobald die Zugangsbeschränkungen aufgehoben sind, auf dem Internet veröffentlicht. 
erster Linie wird sich der Ausschuss für Handel und Entwicklung der WTO mit dieser Frage befassen. Dieser verabschiedete am 15. Oktober 1996 einige Richtlinien, aus denen hervorgeht, dass der Ausschuss selbst die Modalitäten zur Durchführung festsetzt. Die Bemühungen des Generalsekretariats im Bereich der technischen Zusammenarbeit unterliegen ebenfalls diesen Bestimmungen und werden durch den Ausschuss überprüft. Die Modalitäten der Zusammenarbeit wurden am 25. Juli 1997 veröffentlicht. Prinzipiell muss die Zusammenarbeit den Bedürfnissen der Empfängerländer entsprechen. Sie kann verschiedene Formen annehmen: Allgemeine Seminare, Seminare und Workshops über spezifischere Themen, technische Missionen, Praktika, praxisorientierte Ausbildungsprogramme, Schulungsunterlagen, Weitergabe von handelsrelevanten Daten und WTO-Dokumenten sowie Teilnahme an Projekten zur Zusammenarbeit anderer Institutionen. Von besonderer Bedeutung erscheint dabei die Notwendigkeit, die Tätigkeiten mit anderen Institutionen wie dem Internationalen Handelszentrum (International Trade Centre, ITC), der UNCTAD und der Weltbank und, auf besonderen Gebieten wie dem geistigen Eigentum, der WIPO zu koordinieren.

Um die Auswirkungen der Uruguay-Runde auf die Entwicklungsländer besser abschätzen zu können, gab der Bundesrat 1994 einer unabhängigen Instanz den Auftrag, eine Studie durchzuführen. ${ }^{23}$ Diese Studie diente neben den von der Bundesverwaltung durchgeführten Arbeiten als Grundlage für gewisse Massnahmen. Die wichtigste davon ist die Verabschiedung eines Gesetzes und eines Bundesbeschlusses über das allgemeine Präferenzsystem. Ausserdem organisierte der Bund das Seminar von Ancona, an dem sämtliche betroffenen Organisationen, darunter die UNCTAD, das UNDP und das ITC, teilnahmen. Dabei kam auch das geistige Eigentum zur Sprache. Daneben fanden noch weitere wichtige Anlässe statt. Das Ministertreffen über die Massnahmen zur Verbesserung des Marktzugangs für die am wenigsten entwickelten Länder (27.-28. Oktober 1997 am Sitz der WTO in Genf) bildete den vorläufigen Abschluss. Von besonderer Bedeutung an diesem letzten Treffen ist, dass sich manche Entwicklungsländer, deren Industrialisierung bereits ein gewisses Niveau erreicht hat, dazu verpflichtet haben, die LDCs zu unterstützen.

\subsection{ABKOMMEN ZWISCHEN DER SCHWEIZ UND VIETNAM: EINE NEUE GENERATION VON VERTRÄGEN}

Vietnam kämpft gleichzeitig mit den Problemen eines Entwicklungslandes und denjenigen eines Reformstaates, der sich während langer Zeit gegenüber marktwirtschaftlichen Einflüssen abgeschottet hatte. Mittlerweile ist Vietnam für die Schweiz zu einem Schwerpunktland geworden.

Am 20. Oktober 1997 wurde ein «Abkommen zwischen der Schweizerischen Eidgenossenschaft und der Sozialistischen Republik Vietnam über den Schutz des geistigen Eigentums und über die Zusammenarbeit auf dem Gebiet des geistigen Eigentums » paraphiert. Dieses Abkommen, das noch von beiden Parteien endgültig verabschiedet werden muss, stellt eine Premiere dar. Zwar ist das geistige Eigentum bereits in den Freihandelsabkommen der Europäischen Frei-

23. «Effets de l'Uruguay Round sur les pays en développement: Une analyse d'évaluations réalisées à ce jour», Paul Dembinski/Jacques Forster/Jacques Degbelo, Eco’diagnostic-IUED, 1995. 
handelsassoziation mit Drittstaaten und in den bilateralen Verträgen der Schweiz mit zahlreichen Staaten weitgehend berücksichtigt, aber noch nie wurde ein Vertrag ausschliesslich zu dieser Frage abgeschlossen. Die genannten Übereinkommen decken zwar alle das Gebiet der technischen Zusammenarbeit ebenfalls ab, aber in diesem neuen Vertragswerk ist sie eines der beiden Hauptthemen und wird im Vertrag selbst durch ein umfassendes und ausdrückliches Engagement der Schweiz gegenüber Vietnam ergänzt. Der zweite Teil bezieht sich auf die Schutznormen und geht dabei insbesondere auf die folgenden Punkte ein: Einhaltung des Prinzips der Nichtdiskriminierung auf beiden Seiten (Inländerbehandlung und Meistbegünstigungsklausel), und das Engagement Vietnams, innerhalb von zwei Jahren (bis zum Jahr 2000) sein Schutzniveau im Bereich des geistigen Eigentums den Vorgaben des TRIPS-Abkommens anzugleichen. Sollte das Land ausserstande sein, den Termin im Jahr 2000 einzuhalten, kann diese Frist verlängert werden. Die Haltung der Schweiz bei den Verhandlungen war von Realismus geprägt, denn bei dem Beitritt zur WTO handelt es sich um einen "package deal », und es ist duchaus möglich, dass das Stichdatum im Jahr 2000 in manchen Bereichen nicht eingehalten werden kann. Deshalb musste Vietnam ein gewisser Spielraum und ein gewisses Mass an Flexibilität eingeräumt werden. Um Vietnam bei der Verwirklichung dieser Ziele zu unterstützen (die sich das Land bei seinem Beitritt zur WTO übrigens selber gesetzt hat), hat sich die Schweiz zu einem Programm auf dem Gebiet der technischen Zusammenarbeit verpflichtet, welches sich vorläufig über einen Zeitraum von drei Jahren ab dem Inkrafttreten des Abkommens erstreckt. Eine vorläufige Liste mit Aufgaben, die aufgrund der Wünsche Vietnams zusammengestellt wurde, präzisiert den Inhalt des Programms.

\section{Unterstützung bei der Verbesserung}

des Rechtsschutzes auf dem Gebiet der geistigen Eigentumsrechte:

- Studium der massgeblichen internationalen Verträge, insbesondere des TRIPS-Abkommens und gewisser von der WIPO verwalteter Übereinkommen, denen Vietnam noch nicht beigetreten ist; von Fall zu Fall Ausarbeitung von Vorschlägen und Empfehlungen an die zuständigen Behörden, die Frage nach dem Beitritt Vietnams zu diesen Verträgen und Übereinkommen zu prüfen;

- Untersuchung jener Gebiete des geistigen Eigentums, auf denen in Vietnam noch kein Schutzsystem besteht, z.B. geheime Informationen und Bekämpfung des unlauteren Wettbewerbs;

- Abklärung des Modernisierungsbedarfs im rechtlichen Bereich, Unterbreitung von Vorschlägen über neue Gesetze zum Schutz der geistigen Eigentumsrechte oder über Änderungen oder Revisionen bereits bestehender Gesetze, damit Vietnam die Anforderungen der international geltenden Normen und Abkommen, insbesondere des TRIPS-Abkommens (Bestimmungen zum materiellen Recht und zur Durchführung), erfüllen kann.

\section{Unterstützung der für das geistige Eigentum zuständigen Behörden:}

- Erfahrungsaustausch im Verwaltungsbereich zwischen den Behörden der Schweiz und anderer Länder oder Regionen durch Studienreisen, Seminare etc.; 
- Qualifizierte Ausbildung des Personals der für geistiges Eigentum (gewerbliches Eigentum, Urheberrechte und verwandte Schutzrechte) zuständigen Behörden, d.h. Durchführung kurz- und langfristiger Ausbildungsgänge für Führungskräfte, Juristen, Prüfer, Experten im Patentrecht und auf anderen Gebieten und für technische Mitarbeiter, im Rahmen von Seminaren, Symposien und Workshops;

口 Unterstützung bei der Modernisierung der zuständigen Ämter sowie der evtl. vorhandenen regionalen Zweigstellen;

- Modernisierung der Informationssysteme im Patentbereich und auf anderen Gebieten, einschliesslich der Schaffung eines Netzes nationaler Informationsstellen im Bereich des geistigen Eigentums;

口 Bereitstellung der notwendigen technischen Ausrüstung.

Hilfestellung bei der Ausarbeitung von Gesetzen

über das geistige Eigentum und bei deren effektiven Anwendung:

口 Qualifizierte Ausbildung von Richtern (auch im administrativen Bereich), von Zollpersonal und anderen Personen, die für die Umsetzung der Rechte im Bereich des geistigen Eigentums zuständig sind, im Rahmen von Seminaren, Studienreisen und Workshops;

๑ Bereitstellung der notwendigen technischen Ausrüstung.

\section{Andere Tätigkeiten,}

einschliesslich der Förderung des geistigen Eigentums und dessen Nutzung :

口 Sensibilisierung der Öffentlichkeit für die Notwendigkeit, Innovationen und Neuerungen zu schützen und Fälschung und Piraterie zu bekämpfen, im Rahmen von Seminaren mit Ländern (insbesondere mit Entwicklungsländern), die von dieser Problematik betroffen sind;

- Ermutigung der Unternehmen, der einheimischen Gemeinschaften und des einzelnen, die Rechte des geistigen Eigentums in allen technologischen Bereichen in Anspruch zu nehmen;

- Unentgeltliche Nachforschungen durch die Schweizer Behörden zugunsten vietnamesischer Unternehmen oder Erfinder, die ein Patentgesuch einreichen möchten ;

口 Förderung und Entwicklung der Ausbildung auf dem Gebiet des geistigen Eigentums in Vietnam, wobei die Bedeutung dieses Bereichs für die wirtschaftliche Entwicklung des Landes besonders herausgestellt werden soll.

Bei diesem Programm zur technischen Unterstützung wird strikt nach dem Grundsatz der Komplementarität vorgegangen, um Überschneidungen mit den Bemühungen internationaler Organisationen oder anderer Länder zu vermeiden. Besondere Aufmerksamkeit verdienen dabei die Bestrebungen zur Förderung des geistigen Eigentums bei den einheimischen Gemeinschaften, denn sie stehen zur Zeit im Mittelpunkt der Diskussion um den Schutz überlieferter Kenntnisse und des traditionellen Know-hows. Das schweizerische Projekt will diese Gemeinschaften dazu ermutigen, das derzeitige oder ein zukünftiges System des geistigen Eigentums nach Kräften in ihrem eigenen Interesse zu nutzen und eine offensivere Haltung einzunehmen. Ziel ist es, sie mit dem Umgang mit dem 
Patentrecht, mit den Bestimmungen über den Schutz von Pflanzensorten, geographischer Angaben und Markenzeichen, mit dem Urheberrecht und mit den Geschäfts- und Fabrikationsgeheimnissen vertraut zu machen, damit sie ihre Kenntnisse und künftigen Verbesserungen schützen können und in der Lage sind, diese zu ihrem eigenen Wohl zu nutzen, anstatt sie der Allgemeinheit zu überlassen.

\section{SCHLUSSFOLGERUNGEN}

Die Ergebnisse der Uruguay-Runde, die derzeitigen Bestrebungen und die diversen Projekte der Schweiz zeigen, dass ein verbessertes Schutzsystem durchaus mit den Entwicklungsinteressen vereinbar ist. Das TRIPS-Abkommen ist ein überzeugendes Beispiel: Die Teilnahme der Entwicklungsländer an den Arbeiten des TRIPS-Rates ${ }^{24}$ ist der Beweis für die durchgehende Akzeptanz des Abkommens bei diesen Ländern. Bereits bei der Verabschiedung des TRIPSAbkommens spielten sie eine wichtige Rolle, denn ohne ihre Unterstützung wäre die Ausarbeitung eines multilateralen Mechanismus, der diesen Namen auch wirklich verdient, unmöglich gewesen. Sie haben begriffen, dass handfeste Ergebnisse im Bereich des geistigen Eigentums die Voraussetzung für Verbesserungen auf anderen Gebieten sind, die sie interessieren, wie z.B. beim Marktzugang und im Landwirtschafts- und Textilsektor, um nur zwei Beispiele zu nennen. Die Furcht vor unilateraler Druckausübung ${ }^{25}$ war dabei ein wichtiger Faktor. Entscheidend für den Abschluss der Uruguay-Runde war aber, dass die Entwicklungsländer das Potential dieses multilateralen Systems erkannten.

Die WTO bietet allen ihren Mitgliedsstaaten dieselben Möglichkeiten zur Durchsetzung ihrer Interessen. Dabei könnte der Verdacht aufkommen, dass die Industrieländer ein grösseres Gewicht besitzen. Die Tagungen des TRIPS-Rates beweisen jedoch das Gegenteil: Jene Reform- und Entwicklungsländer, die sich aus eigenem Antrieb vor Ablauf der Übergangsfrist einer Prüfung ihrer Gesetze unterzogen, mussten nicht vor einem Panel erscheinen, wenn ihre Bestimmungen nicht dem TRIPS-Abkommen entsprachen. Auch in den demnächst vom TRIPS-Rat behandelten Fragen (geographische Angaben und Patente) werden die Entwicklungsländer ihren Einfluss geltend machen. Auf dem Gebiet der Biotechnologie ist alles offen: Zur Zeit sind sowohl die Hoffnungen auf eine rasche Erhöhung des Schutzes als auch die Erwartungen jener, die eine Schwächung des Schutzes anstreben, verfrüht. Gewisse Länder werden nicht davor zurückschrecken, ein beliebiges TRIPS-Dossier mit einem anderen Fragenkomplex zu verknüpfen (beispielsweise die Verbindung der geographischen Angaben mit der Landwirtschaft). Die Unterhändler der Schweiz werden in diesen Fragen besonders gefordert sein, geht es doch für sie darum, ein globales Gleichgewicht der Verhandlungsergebnisse zu verwirklichen. In der UruguayRunde ist ihnen dies gelungen, und es bleibt zu hoffen, dass sie auch diesmal Erfolg haben.

24. Damit sind insbesondere die Vorbereitungen für die Verhandlungen über die geographischen Angaben gemeint.

25. Die USA machten mehrfach von der unilateralen Prozedur «Super $301 »$ Gebrauch, die es ihnen erlaubt, Zugeständnisse zurückzuziehen, wenn Grund zur Annahme besteht, dass der Schutz des geistigen Eigentums in einem bestimmten Land unzureichend ist und den Interessen ihrer Unternehmen zuwiderläuft. Erwähnenswert ist, dass in dem Masse, wie die Entwicklungsländer die Vorteile einer Verankerung des geistigen Eigentums im GATT erkannten, die USA Zweifel am Nutzen des TRIPS-Abkommens für ihre Wirtschaft zu hegen begannen. 
Ein anderer wichtiger Aspekt ist die Solidarität unter den Staaten. Das Projekt zur Zusammenarbeit der Schweiz mit Vietnam stellt für die Schweizer Behörden einen Testlauf dar. Es geht darum, zu beweisen, dass sich die Schweiz noch stärker engagieren kann. Deshalb muss sie ein Land mit zahlreichen Problemen dabei unterstützen, die Schwelle zur Entwicklung zu überschreiten. Dazu müssen im multilateralen Handelssystem und im TRIPS-Abkommen zahlreiche Anpassungen vorgenommen werden. Zu Zeiten der Globalisierung und der Delokalisierung stellt ein Land mit soliden rechtlichen Rahmenbedingungen ein Magnet für neue Investitionen dar. Zwar ist der Schutz des geistigen Eigentums keine Garantie für den Zustrom von Direktinvestitionen, aber es ist unbestritten, dass er zu einer Verbesserung der Chancen beiträgt. Der derzeitige Wohlstand und Frieden in der Schweiz beruht auf einem Zusammenspiel zahlreicher Faktoren. Einer davon ist der wirtschaftliche Aufschwung, der unter anderem auf dem geistigen Eigentum beruht. Eine Innovation zieht neue Innovationen nach sich. Die Schweiz hat bereits Generationen von Erfindern und Forschern hervorgebracht, die sie zu dem gemacht haben, was sie heute trotz einiger Schwierigkeiten ist: Ein Zentrum für Forschung, Entwicklung und Produktion. Über die technische Zusammenarbeit kann die Schweiz auch andere Nationen an ihren Erfahrungen teilhaben lassen.

QUELLEN

«Botschaft zur Genehmigung der GATT/WTO-Übereinkommen (Uruguay-Runde)» (GATT-Botschaft 1) vom 19. September 1994, Bbl 1994 IV S. 1 ff.

Croone, John, Reshaping the World Trading System. A history of the Uruguay Round, World Trade Organization, 1995.

Cottier, Thomas, "The Prospects for Intellectual Property in GATT», in: Common Market Law Review 28: 383-414, 1991.

Cottier, Thomas \& Tran Thi Thu-Lang, «Le GATT et l'Uruguay Round: l'importance du projet d'accord TRIPS et son impact sur le droit de la propriété intellectuelle», in: Aktuelle Juristische Praxis/Pratique juridique actuelle (AJP/PJA) 5/93, Bern.

Girard, Pierre-Louis, «De Punta del Este à Marrakech: le processus de négociation 1986- 1993 », in: GATT-Uruguay Round, Thomas Cottier (ed.), 1995.

Haeberli, Christian, «Das GATT und die Entwicklungsländer», in: GATT-Uruguay Round, Thomas Cottier (ed.), 1995.

Otten, Adrian, «Improving the Playing Field for Exports: the Agreements on Intellectual Property », Investments Measures and Gvernment Procurement, in: GATT-Uruguay Round, Thomas Cottier (ed.), 1995.

Tran Thi Thu-Lang, «La propriedad intelectual en la industria y el comercio: La globalizaciòn y la funciòn de la propiedad intelectual en la transferencia de tecnologìa y la inversiòn extranjera directa » (doc. OMPI/ACAD/S/96/8), WIPO, Genf, Juni 1996.

UNCTAD Secretariat, The TRIPS Agreement and Developing Countries, 1997. 